\title{
APPROXIMATE TORUS FIBRATIONS OF HIGH DIMENSIONAL MANIFOLDS CAN BE APPROXIMATED BY TORUS BUNDLE PROJECTIONS
}

\author{
BY
}

R. E. GOAD

\begin{abstract}
In this paper, we prove that approximate torus fibrations of high dimensional manifolds can be approximated by torus bundle projections. The principal tools are the torus trick developed by Kirby and Siebenmann, a surgery theorem concerning homotopy structures on torii due to Hsiang and Wall, a theorem on the space of homeomorphisms of the torus due to Hamstrom and a generalization of hereditary homotopy equivalence developed by the author.
\end{abstract}

An old question in topology is how to identify a product or local product structure in a space. Many results have been obtained which tell whether a continuous function is the projection of a local product (fibre bundle) or may be approximated by one. Most of these criteria involve some sort of regularity condition. That is, nearby fibres must lie in the domain in somewhat similar fashion. With only weak hypotheses on the spaces involved, a fairly strong form of regularity (topological) produces a product theorem [D-H]. Chapman and Ferry show that this criterion is satisfied under a "homotopy regularity" condition (that of being an ANR fibration with $Q$-manifold fibres ([C-F 1], [C-F 2])) and so obtain a product theorem for nicer spaces with a weaker regularity hypothesis. Coram and Duvall have shown that an approximate fibration possesses a still weaker form of regularity (shape) called complete movability [C-D 2].

The problem we consider here is whether, for sufficiently nice spaces, such a map may be approximated by a fibre bundle projection.

This paper is divided into two parts. In the first, we develop many of the tools which will be used in the second. These deal, generally, with various constructions and a characterization of approximate fibrations. Some of the details are omitted, but these can be found in the author's Ph. D. thesis [Go] and, hopefully, in a forthcoming paper.

ACKNOWLEDGEMENTS. I would like to take this opportunity to thank my advisor, Professor John Hollingsworth, for many helpful consultations during the development of this work and Professor William Haver for pointing out the 1965 paper of Mary-Elizabeth Hamstrom [Ha] on which this paper turns.

To simplify the exposition, we will deal only with separable metric spaces and, when convenient, we will restrict our attention to proper maps, i.e. those for which the inverse image of a compact set is compact.

Received by the editors March 22, 1978. This work was originally presented to the 1976 Georgia Topology Conference at the University of Georgia, Athens, Georgia, in August, 1976.

AMS (MOS) subject classifications (1970). Primary 57A99, 55F65. 
I. Local homotopy properties. An approximate fibration is a proper map of metric spaces which has the approximate homotopy lifting property with respect to all metric spaces. That is, any commutative diagram

extends to a diagram
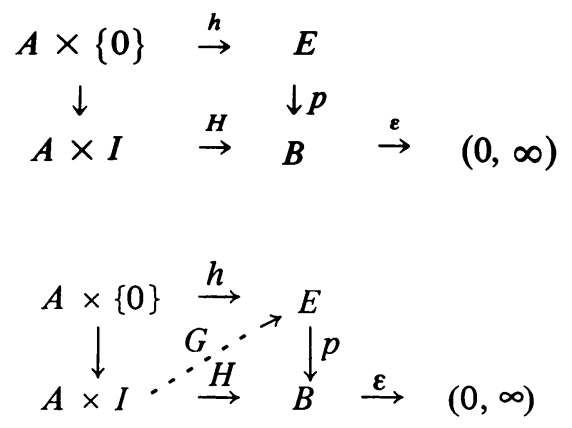

so that $G(a, 0)=h(a, 0)$ and $d(p G(a, i), H(a, i))<\varepsilon H(a, i)$ for all $(a, i)$ in $A \times I$. Although Coram and Duvall have defined the term in a more general setting using open covers rather than a metric [C-D 1], this definition suffices in our context.

A proper map $p: E \rightarrow B$ has local homotopy product structure with fibre $F$ (LHP- $F$ ) if for each $b \in B$, there is a neighborhood $U$ of $b$ and $\operatorname{map} f: p^{-1}(U) \rightarrow$ $F$ so that, for each neighborhood $V$ of $b$ in $U, p \times f: p^{-1}(V) \rightarrow V \times F$ is a proper homotopy equivalence. $p$ has HP- $F$ if we may take $U=B$ in the definition of LHP- $F$. A useful theorem is the following:

I.A. THEOREM. If $p: E \rightarrow B$ is a proper map of $A N R$ 's and there is $a b$ in $B$ so that $p^{-1}(b)$ has the shape of a compact $A N R F$, then $p$ is an approximate fibration if and only if $p$ has property $L H P-F$.

Proof. In case $p$ is an approximate fibration, there is an exact sequence of shape groups [C-D 1]. The shape isomorphism of the fibre with the ANR $F$ provides a map $s$ defined on a neighborhood $U^{\prime}$ of $p^{-1}(b)$. Choose a neighborhood $U$ of $b$ so that $p^{-1}(U) \subseteq U^{\prime}$. This is to be the $U$ required by property LHP- $F$. Now, the sequence is still exact if the map is restricted to $p: p^{-1}(U) \rightarrow U$ and the shape map provides a splitting:

$$
\rightarrow \operatorname{Sh}_{k}(F) \underset{S_{*}}{\rightarrow} \operatorname{Sh}_{k}\left(p^{-1}(U)\right) \stackrel{p *}{\rightarrow} \operatorname{Sh}_{k}(U) \rightarrow \operatorname{Sh}_{k-1}(F)
$$

Further, since all spaces involved are ANR's, shape groups may be replaced by homotopy groups. This splitting provides isomorphisms

$$
\left.P_{*} \oplus S_{*}: \pi_{k}\left(p^{-1}(U)\right) \rightarrow \pi_{k}(F) \oplus \pi_{k}(U)\right) \text {. }
$$

Thus, by the Whitehead theorem, $p \times s$ is a homotopy equivalence. The argument works equally well for $V \subseteq U$ and so it is easily seen, say, via a proper Whitehead theorem [F-T-W], that $p \times s$ is a proper homotopy equivalence of $p^{-1}(V)$ to $V \times F$ for each open $V$ contained in $U$.

Conversely, suppose $p$ has property LHP-F. It is easily seen how to $\varepsilon$-lift the homotopy over any one of the $U$ 's for which the map is homotopically trivial. To 
obtain a global lifting, one simply mimics the induction argument used to show that a coordinate bundle is a (Hurewicz) fibration [St].

We are now free to work with LHP- $F$ maps rather than approximate fibrations whenever it seems more convenient. We will build on the analogy between LHP-F maps and fibre bundles to obtain the tools we will need for approximations. Many of these tools are founded on the following theorem, which has appeared whenever one wished to obtain global homotopy information from local information ([Ko], [Mi] and [Su]).

I.B. TheOREM. If $F$ is a closed $A N R$ subspace of the $A N R E$ and $U=\left\{U_{j}\right\}_{j=1}^{\infty}$ is a locally finite open cover of $E$ such that for each nonempty finite subfamily $S$ of $U$ there is a strong deformation retraction of $U_{S}=\cap \cup_{U_{i} \in S} U_{i}$ onto $F \cap U_{S}$, then there is a strong deformation retraction of $E$ onto $F$ which is limited by $U$.

Proof. The proof given by Milnor in [Mi] actually yields this result. One must observe that the deformation he constructs is limited by $U$.

The next corollary follows routinely from I.B. by use of the mapping cylinder of $p \times f:$

I.B.1. Corollary. Suppose $X, Y$ and $F$ are $A N R$ 's, $p: X \rightarrow Y, f: X \rightarrow F$ are maps and $\left\{U_{i}\right\}$ is a locally finite open cover of $Y$ such that, for every $U_{i}, p: p^{-1}\left(U_{i}\right) \rightarrow U_{i}$ has property $H P-F$ via $f$. Then $p: X \rightarrow Y$, has property $H P-F$ via $f$. Further, we may find a homotopy inverse $g$ for $p \times f$ so that $g\left(U_{i} \times F\right) \subseteq p^{-1}\left(U_{i}\right)$ and homotopies

$$
h: g \circ(p \times f) \simeq 1_{X}, \quad k:(p \times f) \circ g \simeq 1_{Y \times F}
$$

so that $h\left(p^{-1}\left(U_{i}\right) \times I\right) \subseteq p^{-1}\left(U_{i}\right)$ and $k\left(U_{i} \times F \times I\right) \subseteq U_{i} \times F$.

I.B.2. CoRollary. If $p$ has property $H P-F$ via $f: X \rightarrow F$ and $\alpha: B \rightarrow Y$ is an open immersion, then the topological pullback $p^{\prime}$ of $p$ by $\alpha$ has property $H P-F$ via $f \circ \alpha^{\prime}$ :

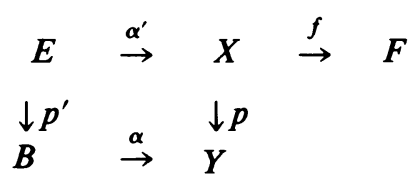

Proof. The open sets openly embedded by $\alpha$ clearly have HP- $F$ via $f \circ \alpha^{\prime}$. A locally finite refinement of this cover provides the necessary cover of $\boldsymbol{B}$.

The next proposition provides a means to alter HP trivializations:

I.C. Proposition. Suppose $p: X \rightarrow Y$ has property $H P-F$ via $f: X \rightarrow F$. If $H$ is a homotopy of $f$ to $f^{\prime}$, then $P$ has $H P-F$ via $f^{\prime}$.

Proof. $1 \times H$ is a proper homotopy of $p \times f$ to $p \times f^{\prime}$, even when restricted to $p^{-1}(U)$.

One can glue HP trivializations according to the following proposition: 
I.D. Proposition. Suppose $p: X \rightarrow Y$ has property $H P-F$ via $f: X \rightarrow F$, and that $H$ is a homotopy from $f$ to $f^{\prime}$. If $B_{1}$ and $B_{2}$ are two disjoint closed subsets of $X$, then there is a third map $\mathrm{g}: X \rightarrow F$ such that

(1) $p$ has $H P-F$ via $g$, and

(2) $g\left|B_{1}=f\right| B_{1}$ and $g\left|B_{2}=f^{\prime}\right| B_{2}$.

Proof. Choose a Urysohn function $u: X \rightarrow[0,1]$ such that $u\left(B_{1}\right)=0$ and $u\left(B_{2}\right)=1$. Set $g(x)=H(x, u(x))$. If $x$ is in $B_{1}$, then $g(x)=H(x, 0)=f(x)$ and if $x$ is in $B_{2}$, then $g(x)=H(x, 1)=f^{\prime}(x)$. Thus condition (2) above is satisfied. To verify condition (1) we must show that $g$ is homotopic to $f$. Set $L(x, t)=H(x,(1-$ $t) u(x))$. Note that $L(x, 0)=H(x, u(x))=g(x)$ and that $L(x, 1)=H(x, 0)=f(x)$.

The next proposition allows one to combine HP trivializations which do not necessarily agree on their common domain. This facilitates certain pasting constructions which are necessary to the approximation scheme.

To establish notation, suppose $p: X \rightarrow Y$ is a map and that $Y$ is the union of two open sets $A_{1}$ and $A_{2}$. Set

$$
\begin{aligned}
B_{1}^{\prime}=Y \backslash A_{2}, \quad B_{2}^{\prime}=Y \backslash A_{1}, \\
C_{1}^{\prime}=\left\{y \in A_{1} \cap A_{2}: 2 d\left(y, B_{1}^{\prime}\right)<d\left(y, B_{2}^{\prime}\right)\right\}, \\
C_{2}^{\prime}=\left\{y \in A_{1} \cap A_{2}: 2 d\left(y, B_{2}^{\prime}\right)<d\left(y, B_{1}^{\prime}\right)\right\}, \\
C_{i}=p^{-1}\left(C_{i}^{\prime}\right), \quad B_{i}=p^{-1}\left(B_{i}^{\prime}\right) ; \quad i=1,2, \\
D_{1}^{\prime}=\left\{y \in Y: 2 d\left(y, B_{1}^{\prime}\right)<d\left(y, B_{2}^{\prime}\right)\right\}, \\
D_{2}^{\prime}=\left\{y \in Y: 2 d\left(y, B_{2}^{\prime}\right)<d\left(y, B_{1}^{\prime}\right)\right\}, \\
D_{i}=p^{-1}\left(D_{i}^{\prime}\right) .
\end{aligned}
$$

I.E. Proposition. For $i=1,2$ suppose $p \mid p^{-1}\left(A_{i}\right): p^{-1}\left(A_{i}\right) \rightarrow A_{i}$ has property $H P-F$ via some $f_{i}$. If $f_{1}$ is homotopic to $f_{2}$ on $p^{-1}\left(A_{1} \cap A_{2}\right)$, then $p$ has property $H P-F$ via a map $g: X \rightarrow F$ such that, for $i \in\{1,2\}, g\left|B_{i}=f_{i}\right| B_{i}$.

Proof. We first show that $C_{1}^{\prime} \cap C_{2}^{\prime}=\varnothing$ :

Let $y$ be an element of $C_{1}^{\prime} \cap C_{2}^{\prime}$, then $2 d\left(y, B_{1}^{\prime}\right)<d\left(y, B_{2}^{\prime}\right)<d\left(y, B_{1}^{\prime}\right)$, so $d\left(y, B_{1}^{\prime}\right)=0$. by the same argument, $d\left(y, B_{2}^{\prime}\right)=0$ and so $y \in B_{1}^{\prime} \cap B_{2}^{\prime}$. Thus $C_{1}^{\prime} \cap C_{2}^{\prime} \subseteq B_{1}^{\prime} \cap B_{2}^{\prime}$. However, since $Y=A_{1} \cup A_{2}$,

$$
B_{1}^{\prime} \cap B_{2}^{\prime}=\left(Y \backslash A_{2}\right) \cap\left(Y \backslash A_{1}\right)=Y \backslash\left(A_{1} \cup A_{2}\right)=\varnothing .
$$

Thus, $B_{1} \cap B_{2}=\varnothing$ and $C_{1} \cap C_{2}=\varnothing$. By I.D., there is a function $g^{\prime}: p^{-1}\left(A_{1} \cap\right.$ $\left.A_{2}\right) \rightarrow F$ which agrees with $f_{i}$ on $C_{i}$ and which is an HP trivialization for $p \mid p^{-1}\left(A_{1}\right.$ $\left.\cap A_{2}\right)$. Now, observe that each $D_{i}$ is an open neighborhood of $B_{i}$ and that $D_{i} \cap p^{-1}\left(A_{1} \cap A_{2}\right) \subseteq C_{i}$. Again, note that $D_{1}$ and $D_{2}$ are disjoint because $D_{i} \subseteq B_{i}$ $\cup C_{i}$. Thus $\left\{D_{1}, D_{2}, p^{-1}\left(A_{1} \cap A_{2}\right)\right\}$ is an open cover of $X$ with the properties that $D_{1} \cap D_{2}=\varnothing$, and $D_{i} \cap p^{-1}\left(A_{1} \cap A_{2}\right) \subseteq C_{i}$. 
Thus, setting

$$
g(x)= \begin{cases}f_{1}(x) & \text { if } x \in D_{1}, \\ g^{\prime}(x) & \text { if } x \in p^{-1}\left(A_{1} \cap A_{2}\right), \\ f_{2}(x) & \text { if } x \in D_{2}\end{cases}
$$

we obtain a continuous function, since each pair of the three component functions agree on their common domains. Thus, by I.B.1, $p$ is HP-F via $g$. Note that since $B_{i} \subseteq D_{i}$, we have that $g\left|B_{i}=f_{i}\right| B_{i}$ for $i \in\{1,2\}$.

II. The approximation scheme. The principal result is the following:

II.A. TheOREM. Suppose $p: M^{n+2} \rightarrow N^{n}$ is an LHP-T $T^{2}$ map of topological manifolds and $n \geqslant 6$. Suppose that $\varepsilon: N^{n} \rightarrow(0, \infty)$ is continuous. Then there is a torus bundle projection $p^{\prime}: M \rightarrow N$ with the property that, for every $m$ in $M$, $d\left(p(M), p^{\prime}(M)\right)<\varepsilon(p(m))$.

Here the term bundle or fibre bundle denotes a (locally trivial) fibre bundle in the sense of Steenrod [St] and the transformation group of the fibre is the group of homeomorphisms of the fibre. Also, we will use the term "over" to mean "when restricted to the inverse image of". For example, to say " $p$ has HP- $T^{2}$ over $U$ " means $p \mid p^{-1}(U): p^{-1}(U) \rightarrow U$ has property HP- $T^{2}$.

Theorem II.A depends on:

II.B. THEOREM. Let $p: V^{m+2} \rightarrow B^{k} \times R^{n}, k+n=m>6$, be an HP-T map of topological manifolds of the indicated dimensions. If $p$ is a trivial $T^{2}$-bundle projection over the neighborhood $\left(B^{k} \backslash \frac{1}{2} \dot{B}^{k}\right) \times R^{n}$ of $\partial B^{k} \times R^{n}$, then there is an $H P-T^{2}$ map $p^{\prime}: V^{m+2} \rightarrow B^{k} \times R^{n}$ such that

(1) $p^{\prime}$ is a (trivial) $T^{2}$-bundle projection over $B^{k} \times B^{n}$ and

(2) $p^{\prime}=p$ over $\left(\partial B^{k} \times R^{n}\right) \cup\left[B^{k} \times\left(R^{n} \backslash r \dot{B}^{n}\right)\right]$ for some radius $r$.

II.B depends on:

II.C. LEMMA. Given $p, V, m, k, n$ as above, there are maps $\phi: V \rightarrow B^{k} \times R^{n} \times T^{2}$ and $F: B^{k} \times R^{n} \times T^{2} \rightarrow B^{k} \times R^{n}$ such that $\phi$ is a homeomorphism, $F$ has property $H P-T^{2}$ and

(1) $F=$ projection to $B^{k} \times R^{n}$ over $\left(\left(B^{k} \backslash r B^{k}\right) \times R^{n}\right) \cup\left(B^{k} \times\left(R^{n} \backslash 4 B^{n}\right)\right.$ for some $r<1$ and

(2) $F \phi=p$ over $\left(\partial B^{k} \times R^{n}\right) \cup\left(B^{k} \times B^{n}\right)$.

Notice that II.C is essentially II.B except that the roles of 0 and $\infty$ in the $R^{n}$ factor are reversed. II.A follows from II.B using induction and an easy computation. II.C is proved using a modification of the main diagram argument used by Siebenmann in [Si 1]. 
Thus, we will prove II.A, II.B, and II.C in reverse order.

The outline of this proof is the same as that found in [Si 1] and we will assume that the reader is somewhat familiar with the "main diagram" ([Ki], [K-S] and [Si 1]).

Proof OF II.C. The proof of II.C is based on constructing the following version of the main diagram:

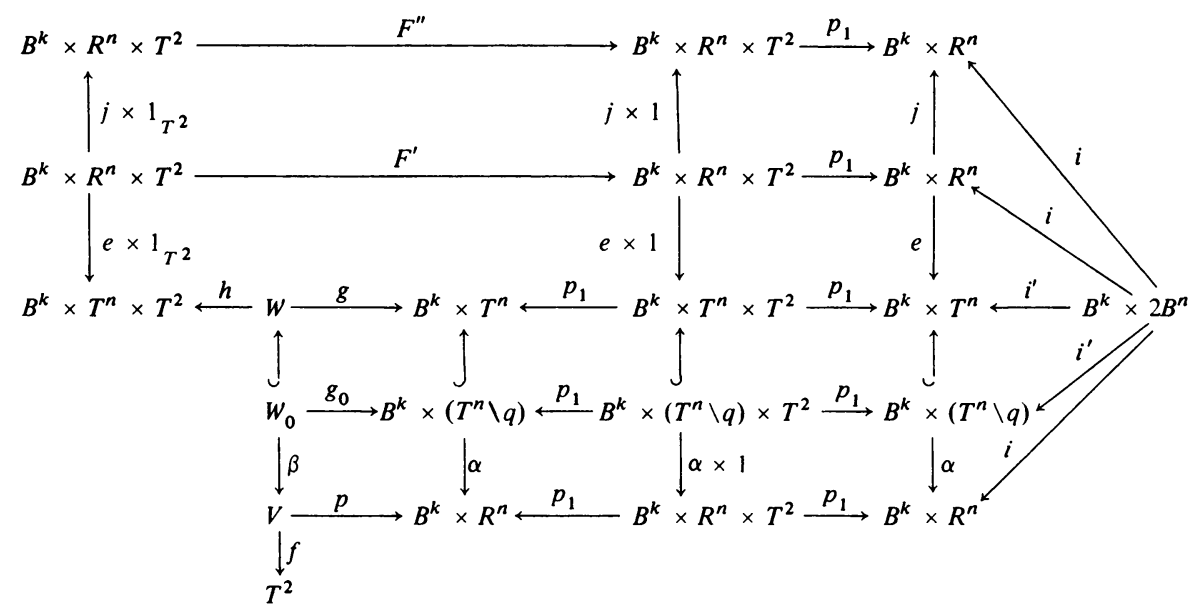

(a) As in [Si 1], $i$ is inclusion, $\bar{e}$ is an exponential map which is 1 to 1 on the interior of each $8 \times 8$ square, $e$ is $1_{B^{k}} \times \bar{e}, q$ is a point not in $\bar{e}\left(2 B^{n}\right)$ and $e i=i^{\prime} . j$ is the embedding constructed in [Si 1], and $\alpha=1 \times \bar{\alpha}$ where $\bar{\alpha}$ is an immersion of $T^{n} \backslash q$ in $R^{n}$.

(b) We wish to find a map $f: V \rightarrow T^{2}$ that is an HP trivialization for $p$ and that is also a bundle trivialization over $\left(B^{k} \backslash \frac{1}{2} \dot{B}^{k}\right) \times R^{n}=N$. First take any bundle trivialization $T: p^{-1}(N) \rightarrow N \times T^{2}$ and any LHP trivialization $f_{1}: V \rightarrow T^{2}$.

We have the following diagram:

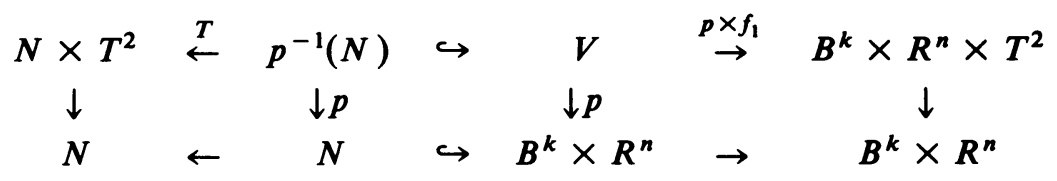

We wish to extend $T$ to all of $V$; however, there are certainly trivializations which will not extend. We therefore modify $T$ as follows: Consider $\left(p \times f_{1}\right) \circ T^{-1}$. This is a fibre homotopy trivialization of the bundle $N \times T^{2}$ over $N$ and so it is fibre homotopic to a genuine bundle trivialization $T^{\prime}$. Let $g: B^{k} \times R^{n} \times T^{2} \rightarrow E$ be a homotopy inverse to $p \times f$ which also restricts to a homotopy inverse to $p \times$ $f \mid p^{-1}(N)$. We have the following homotopy commutative diagram: 


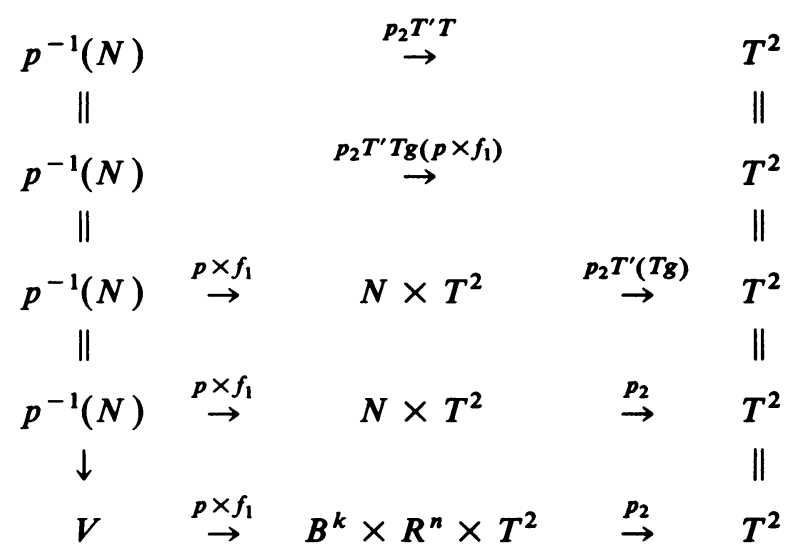

The bottom row is an extension of the previous row over all of $V$. Thus, $T^{\prime} \circ T$ admits an extension $f: V \rightarrow T^{2}$. Since $f \mid p^{-1}(N)=T^{\prime} \circ T$, it is a bundle trivialization.

It remains to show that $f$ is an HP trivialization for $p: V \rightarrow B^{k} \times R^{n}$. However, since $p: V \rightarrow B^{k} \times R^{n}$ has property HP- $T^{2}$, there is a strong deformation retraction of $V$ to the fibre over any point in $\partial B^{k} \times R^{n}$. Since $f$ is certainly a homotopy equivalence on this fibre, it must be a homotopy equivalence on all of $V$.

(c) Let $W_{0}$ be the topological pullback of

$$
\begin{array}{lcl} 
& B^{k} \times T^{n} \backslash q \\
& & \downarrow \alpha \\
V \quad \stackrel{p}{\rightarrow} & B^{k} \times R^{n}
\end{array}
$$

and let $g_{0}, \beta$ be the projections. These have the properties that $\beta$ is an immersion [Si 1, Lemma 2.3] and so $W_{0}$ is a manifold. Since $\alpha$ is a codimension 0 , hence open, immersion, we have that $g$ has property HP- $T^{2}$ via $f \circ \beta$. Note also that, on $g_{0}^{-1} \alpha^{-1}(N), g_{0}$ is a fibre bundle projection and $f \circ \beta$ is a bundle trivialization.

(d) To construct $g: W \rightarrow B^{k} \times T^{n}$, we first construct $g_{1}: W_{1} \rightarrow\left(B^{k} \times T^{n}\right) \backslash$ $\left(\frac{1}{2} B^{k} \times q\right)$. To do this, let

$$
N_{0}=\left(B^{k} \backslash \frac{1}{2} B^{k}\right) \times\left(T^{n} \backslash q\right)=\alpha^{-1}(N) .
$$

Now, $g_{0} \times(f \circ \beta): g_{0}^{-1}\left(N_{0}\right) \rightarrow N_{0} \times T^{2}$ is a homeomorphism. Note that

$$
N_{0} \times T^{2} \subseteq\left(B^{k} \backslash \frac{1}{2} B^{k}\right) \times T^{n} \times T^{2} .
$$

Thus, if we use $g_{0} \times(f \circ \beta)$ as an attaching map, we obtain a manifold

$$
W_{1}=W_{0} \cup\left(B^{k} \backslash \frac{1}{2} B^{k}\right) \times T^{n} \times T^{2}
$$

which maps via $g_{1}$ to $\left(B^{k} \times T^{n}\right) \backslash\left(\frac{1}{2} B^{k} \times\{q\}\right)$ as follows:

$$
g_{1}(x)=\left\{\begin{array}{l}
g_{0}(x), \quad x \in W_{0}, \\
a, \quad x=(a, z) \in\left(\left(B^{k} \backslash \frac{1}{2} B^{k}\right) \times T^{n}\right) \times T^{2}
\end{array}\right.
$$


Note also that $W_{1}$ maps to $T^{2}$ as follows:

$$
f_{1}(x)=\left\{\begin{array}{l}
f \circ \beta(x), \quad x \in W, \\
z, \quad x=(a, z) \in\left(\left(B^{k} \backslash \frac{1}{2} B^{k}\right) \times T^{n}\right) \times T^{2} .
\end{array}\right.
$$

Note that each of $g_{1}, f_{1}$ is well defined and hence continuous. We have that, over $\left(B^{k} \backslash \frac{1}{2} B^{k}\right) \times T^{n}, f_{1}$ is a bundle trivialization of $g_{1}$, by construction. Over $\left(B^{k}\right.$ $\left.\backslash \frac{1}{2} B^{k}\right) \times\left(T^{n} \backslash q\right), g_{1}$ and $f_{1}$ agree with $g_{0}$ and $f \circ \beta$ respectively. Thus, $g_{1}$ has property HP- $T^{2}$ via $f_{1}$.

Now, to obtain $g: W \rightarrow B^{k} \times T^{n}$, choose $\delta: B^{k} \times T^{n} \rightarrow B^{k} \times T^{n}$ to be a continuous onto map such that

(1) $\delta\left(\frac{1}{2} B^{k} \times\{q\}\right)=\{(0, q)\}$,

(2) $\delta$ pointwise fixes a neighborhood of $e\left(B^{k} \times 2 B^{n}\right)$, and

(3) $\delta$ is a homeomorphism on the complement of $\left(\frac{1}{2} B^{k} \times\{q\}\right)$.

Then set $g_{2}=\delta g_{1}: W_{1} \rightarrow\left(B^{k} \times T^{n}\right) \backslash\{(0, q)\}$ and note that $g_{2}$ has property HP- $T^{2}$ via $f_{1}$ and $g_{2}=g_{0}$ near $e\left(B^{k} \times 2 b^{n}\right)$. We wish to extend $W_{1}$ to a manifold $W$ and extend $g_{2}$ and $f_{1}$ to maps $g: W \rightarrow B^{k} \times T^{n}$ and $f_{2}: W \rightarrow T^{2}$ so that $g$ has property HP- $T^{2}$ via $f_{2}$. In [Si 1], the corresponding problem is solved by taking the one-point compactification of $W_{1}$ and sending $\infty$ to $(0, q)$. Here, however, the problem is more delicate. It requires the addition of an entire fibre to $W_{1}$ in such a way that $f_{1}$ can be extended. Once again, it is the fact that each self homotopy equivalence of $S^{k} \times T^{2}$ is homotopic to a homeomorphism [H-W], which provides the necessary map. For the construction, let $S^{m-1} \times R$ be an annular neighborhood of the deleted point $(0, q)$ in $B^{k} \times T^{n}$ with $(0, q)$ at $+\infty$ and consider $U=g_{2}^{-1}\left(S^{m-1} \times R\right)$. According to property HP-T $T^{2}, g_{2} \times f_{1}: U \rightarrow\left(S^{m-1} \times R\right) \times$ $T^{2}$ is a proper homotopy equivalence. Thus, the ends of $U$ are tame, and $\tilde{K}_{0}\left(\pi_{1}\left(E_{i}\right)\right)=0$ for each end $E_{i}$ of $U$. Thus, according to Siebenmann's thesis [Si 2], there is a manifold $M_{i}$ and a homeomorphism $\zeta_{i}: M_{i} \times[0,1) \rightarrow E_{i}$. Now, $\overline{U \backslash E_{1} \cup E_{2}}$ is an $h$-cobordism of $M_{1}$ to $M_{2}$ and each $M_{i}$ is homotopy equivalent to $S^{m-1} \times T^{2}$ by $g_{2} \times f_{1}$. Since $\mathrm{Wh}(Z \oplus Z)=0$, the $s$-cobordism theorem yields a homeomorphism of $\overline{U \backslash E_{1} \cup E_{2}}$ to $M_{i} \times I$. Thus, there is a homeomorphism $\zeta$ : $U \rightarrow M \times R$. Now the composition

$$
M=M \times\{0\} \rightarrow M \times R \stackrel{\zeta^{-1}}{\rightarrow} U^{g_{2} \times f_{1}} \rightarrow S^{m-1} \times R \times T^{2} \rightarrow S^{m-1} \times T^{2}
$$

is a homotopy equivalence, and by [H-W], it is homotopic to a homeomorphism $\lambda$ : $M \rightarrow S^{m-1} \times T^{2}$. Thus

$$
M \times R \stackrel{\xi^{-1}}{\rightarrow} U \stackrel{g_{2} \times f_{1}}{\rightarrow}\left(S^{m-1} \times R\right) \times T^{2}
$$

is homotopic to $\lambda \times 1_{R}=\lambda^{\prime}$ and, in particular, $f_{1} \zeta^{-1}$ is homotopic to $p_{2} \lambda^{\prime}$ and so $p_{2} \lambda^{\prime} \zeta$ is homotopic to $f_{1}$. Now, define $a: U \rightarrow B^{m} \times T^{2}$ by the composition $U \rightarrow S^{m-1} \times R \times T^{2} \hookrightarrow \stackrel{\circ}{ }^{m} \times T^{2}$ and set $W=W_{1} \cup_{a}\left(\dot{B}^{m} \times T^{2}\right)$. Define $g: W$ $\rightarrow B^{k} \times T^{n}$ by the formula

$$
g(w)= \begin{cases}g_{2}(w), & w \in W_{1}, \\ (0, q), & w=(0, z) \in\{0\} \times T^{2} .\end{cases}
$$


Observe that $g$ is continuous, because $g_{2}$ is proper. We now show that $g$ has property HP- $T^{2}: g \mid \dot{B}^{m} \times T^{2}$ clearly has HP- $T^{2}$ via $p_{2}$ and $g \mid W_{1}$ has HP- $T^{2}$ via $f_{1}$. On $\left(\dot{B}^{m} \times T^{2}\right) \cap W_{1}, p_{2} a=p_{2} \lambda^{\prime} \zeta$ which is homotopic to $f_{1}$ and so there is an HP- $T^{2}$ trivialization $f_{2}$ for $g$ on $W$, which agrees with $f_{1}$ on $W_{1} \backslash U$.

(e) $h: W \rightarrow B^{k} \times T^{n} \times T^{2}$ is a homeomorphism such that $h=g \times f_{2}$ on a neighborhood of $\partial W$ and $h$ is homotopic to $g \times f_{2}$. Note that $g h^{-1}$ has property HP- $T^{2}$ via $f_{2} h^{-1}$ which is homotopic to $p_{2}: B^{k} \times T^{n} \times T^{2} \rightarrow T^{2}$. Thus, $g h^{-1}$ has property HP- $T^{2}$ via $p_{2}$.

(f) $F^{\prime}$ is a covering of $g h^{-1} \times p_{2}$ which fixes $\partial B^{k} \times R^{n} \times T^{2}$. Note that $p_{2} F^{\prime}=p_{2}$ and that, since $g h^{-1} \times p_{2}$ is a homotopy equivalence, the diagram

$$
\begin{array}{ccc}
B^{k} \times R^{n} \times T^{2} & \stackrel{F^{\prime}}{\rightarrow} & B^{k} \times R^{n} \times T^{2} \\
\downarrow e \times 1 & & \downarrow e \times 1 \\
B^{k} \times T^{n} \times T^{2} & \stackrel{g h^{-1} p_{2}}{\rightarrow} & B^{k} \times T^{n} \times T^{2}
\end{array}
$$

is a bundle map and hence a pullback diagram. Thus, $p_{1} F^{\prime}$ has property HP- $T^{2}$ via $p_{2}$.

(g) $F^{\prime \prime}$ is obtained from $F^{\prime}$ in the usual fashion ([Si 1], [K-S], etc.) so that the top rectangle in the diagram commutes, $F^{\prime \prime}$ is the identity off $B^{k} \times 4 B^{n} \times T^{2}$ and $F^{\prime \prime}$ has property HP- $T^{2}$ via $p_{2}$.

This completes the construction of the diagram. To complete the lemma, we must construct $\phi$. Note first, that by moving vertically up the left-hand side of the diagram, one obtains a homeomorphism $\psi$, provided he restricts his attention to $p^{-1}\left(B^{k} \times 2 B^{n}\right) . \phi$ is obtained from $\psi$ by an Alexander isotopy used in conjunction with the collaring of $\partial V$ [Si 1]. This completes the lemma.

For the proof of II.B, we adapt the inversion device of [Si 1] as follows:

Let $\alpha: R^{n} \rightarrow R^{n}$ be the homeomorphism given by $\alpha(x)=4 X$. Then, with the data of II.B, let $\tilde{p}=\left(1_{B^{k}} \times \alpha^{-1}\right) p$, and let $f_{1}=F \phi: V \rightarrow B^{k} \times R^{n}$ be as given by Lemma II.C applied to $\tilde{p}$. $F$ and $\phi$ together provide a manifold $\bar{V}=B^{k} \times S^{n} \times$ $T^{2}$ containing $V$ and an HP- $T^{2}$ map $\bar{f}_{1}: V \rightarrow B^{k} \times\left(R^{n} \cup \infty\right)$ extending $f_{1}$ as follows:

$$
\phi: V \rightarrow B^{k} \times R^{n} \times T^{2} \rightarrow B^{k} \times\left(R^{n} \cup \infty\right) \times T^{2}=B^{k} \times S^{n} \times T^{2}
$$

is an embedding.

Define $\bar{V}=V \cup_{\phi} B^{k} \times S^{n} \times T^{2}$ and define $\bar{f}_{1}: \bar{V} \rightarrow B^{k} \times\left(R^{n} \cup \infty\right)$ according to the rule

$$
\bar{f}_{1}(x)= \begin{cases}F(x) & \text { if } x \in B^{k} \times R^{n} \times T^{2}, \\ p_{1}(x) & \text { if } x \in N_{\delta}\left(B^{k} \times \infty \times T^{2}\right),\end{cases}
$$

where $\delta$ is chosen small enough that $F=p_{1}$ on $N_{\delta}\left(B^{k} \times \infty \times T^{2}\right) \cap V$.

Define $\theta: B^{k} \times\left\{\left(R^{n} \cup \infty\right) \backslash 0\right\} \rightarrow B^{k} \times R^{n}$ according to the rule $\theta(x, y)=$ $\left(x, y /|y|^{2}\right)$ if $y \neq \infty$ and $\theta(x, \infty)=(x, 0)$. Note that $\theta^{-1}\left(B^{k} \times \partial r B^{n}\right)=B^{k} \times$ $\partial\left(r^{-1} B^{n}\right)$. 
Let $g_{1}: \bar{V} \backslash p^{-1}\left(B^{k} \times 0\right) \rightarrow B^{k} \times\left\{\left(R^{n} \cup \infty\right) \backslash 0\right\}$ be the restriction of $\bar{f}_{1}$ and let $\overline{\boldsymbol{g}}_{1}$ be the following composition:

$$
\bar{V} \backslash p^{-1}\left(B^{k} \times 0\right) \stackrel{g_{1}}{\rightarrow} B^{k} \times\left\{\left(R^{n} \cup \infty\right) \backslash 0\right\} \stackrel{\theta}{\rightarrow} B^{k} \times R^{n}
$$

Applying Lemma II.C to $\bar{g}_{1}$, we obtain $\bar{g}_{2}: V \backslash p^{-1}\left(B^{k} \times 0\right) \rightarrow B^{k} \times R^{n}$. Setting $g_{2}=\theta^{-1} \bar{g}_{2}$, we have that $g_{2}$ is a bundle projection over $B^{k} \times\left(\frac{1}{4} B^{n} \backslash 0\right)$ and $g_{2}=g_{1}$ over $B^{k} \times\left\{\left(R^{n} \cup \infty\right) \backslash B^{n}\right\}$ and over $\partial B^{k} \times\left\{\left(R^{n} \cup \infty\right) \backslash 0\right\}$.

We can now define $h: V \backslash p^{-1}\left(B^{k} \times 0\right) \rightarrow B^{k} \times\left(R^{n} \backslash 0\right)$ by setting $h$ equal to $\tilde{p}$ over $B^{k} \times\left(R^{n} \backslash \dot{B}^{n}\right)$ and setting $h$ equal to $g_{2}$ over $B^{k} \times\left(B^{n} \backslash 0\right)$. Then $h$ is well defined and continuous because over $B^{k} \times \partial B^{n}$, one has $g_{2}=g_{1}=\bar{f}_{1}=\tilde{p}$. Clearly $h$ is HP- $T^{2}$ and equals $\tilde{p}$ over $\partial B^{k} \times\left(R^{n} \backslash 0\right)$. Thus we can extend $h$ to

$$
h^{\prime}: V \backslash \tilde{p}^{-1}\left(\frac{1}{2} B^{k} \times 0\right) \rightarrow B^{k} \times R^{n} \backslash \frac{1}{2}\left(B^{k} \times 0\right)
$$

by setting $h^{\prime}=\tilde{p}$ on $\tilde{p}^{-1}\left(\left(B^{k} \backslash \frac{1}{2} B^{k}\right) \times 0\right)$.

Now, consider $h^{\prime-1}\left(\partial\left(B^{k} \times \frac{1}{10} B^{n}\right)\right)$. This is a copy of $S^{m-1} \times T^{2}$ and so, by [H-W] and the $h$-cobordism theorem, it bounds a topological $B^{m} \times T^{2}$.

Thus, the restriction of $h^{\prime}$ to $V \backslash\left(B^{m} \times T^{2}\right)$ extends as follows to an HP-T map $p^{\prime}: V \rightarrow B^{k} \times R^{n}$ :

On $V \backslash\left(\stackrel{\circ}{B}^{m} \times T^{2}\right)$, set $p^{\prime}(x)=h^{\prime}(x)$, and on $B^{m} \times T^{2}$, first project to $B^{m}$ and then cone. $\tilde{p}^{\prime}=\alpha p^{\prime}$ is the approximation to $p$ asserted by II.B.

We obtain II.A from II.B by induction over the index of handles in a small handle decomposition of the range. The details are left to the reader. The only new problem is that one must show that the bundle which has been constructed inductively over the boundary of a $k$-handle is, indeed, trivial. Notice that property HP- $T^{2}$ assures us that it will be fibre homotopically trivial. In the case at hand, that of $T^{2}$ fibres, this is sufficient because the group of homotopy equivalences of $T^{2}$ is homotopy equivalent to the group of homeomorphisms of $T^{2}$ [Ha].

Note that the proof given for $T^{2}$ fibres is sufficient for $T^{k}$ fibres with the exception of the last paragraph. The last paragraph is valid for $T^{1}=S^{1}$ fibres and so the corresponding approximation results hold [Go]. The problem remains substantially open for $k>2$.

ADDED IN PROOF. Recent work of F. S. Quinn provides a sequence of obstructions for approximating with topological block bundle projections. These obstructions all vanish for $T^{n}$. See F. S. Quinn, Ends of maps and applications, Bull. Amer. Math. Soc. 1 (1979), 270-272.

\section{BIBLIOGRAPHY}

[C-D 1] D. S. Coram and P. F. Duvall, Approximate fibrations, Rocky Mountain J. Math. 7 (1977), 275-288.

[C-D 2] __, Approximate fibrations and a movability condition for maps, Pacific J. Math. 72 (1977), 41-56.

[C-F 1] T. A. Chapman and S. Ferry, Hurewicz fiber maps with ANR fibers, Topology 6 (1977), 121-143.

[C-F 2] _ Hurewicz fiberings of ANR's (preprint).

[D-H] E. Dyer and M.-E. Hamstrom, Completely regular mappings, Fund. Math. 45 (1957), 103-118.

[F-T-W] F. T. Farrell, L. R. Taylor and J. B. Wagoner, The Whitehead theorem in the proper category, Compositio Math. 27 (1973), 1-23. 
[Go] R. E. Goad, Local homotopy properties of maps and approximation of fibre bundle projections, Thesis, University of Georgia, 1976.

[Ha] M.-E. Hamstrom, The space of homeomorphisms on a torus, Illinois J. Math. 9 (1965), 59-65.

[H] L. S. Husch, Approximating approximate fibrations by fibrations, Canad. J. Math. 29 (1977), 897-913.

[H-W] W.-C. Hsiang and C. T. C. Wall, On homotopy tori. II, Bull. London Math. Soc. 1 (1969), 341-342.

[Ki] R. C. Kirby, Lectures on triangulation of manifolds, Notes, UCLA, 1969.

[K-S] R. C. Kirby and L. C. Siebenmann, Foundations of topology, Notices Amer. Math. Soc. 16 (1969), 848.

[Ko] George Kozlowski, Variants of homotopy equivalence, contributed lecture, CBMS/NSF Regional Conference on the theory of infinite dimensional manifolds and its applications to topology, October 11-15, 1975, Guilford College, Greensboro, N. C.

[Mi] John Milnor, On spaces having the homotopy type of a CW-complex, Trans. Amer. Math. Soc. 90 (1959), 272-280.

[Si 1] L. C. Siebenmann, Approximating cellular maps by homeomorphisms, Topology 11 (1972), 271-294.

[Si 2] __ The obstruction to finding a boundary for an open manifold of dimension greater than five, Thesis, Princeton University, 1965.

[St] Norman Steenrod, The topology of fibre bundles, Princeton Univ. Press, Princeton, N. J., 1951.

[Su] D. P. Sullivan, Triangulating homotopy equivalences, Thesis, Princeton University, 1966.

[Wa] C. T. C. Wall, Surgery on compact manifolds, Academic Press, New York, 1970.

Department of Mathematics, Sam Houston State University, Huntsville, TeXas 77340

Current address: Department of Mathematics, University of Tennessee, Knoxville, Tennessee 37916 